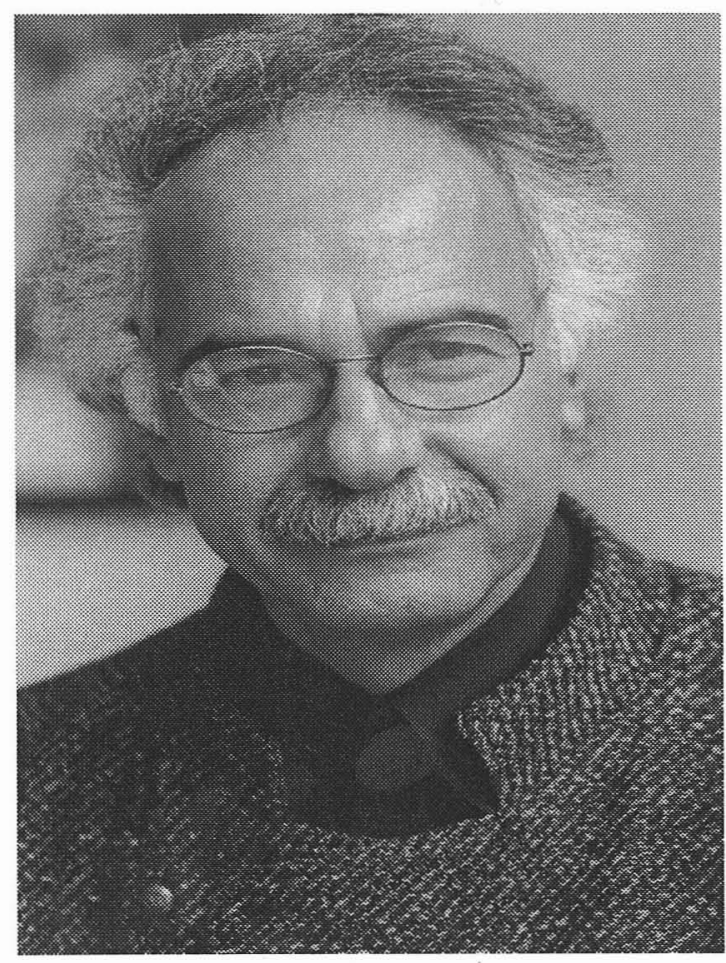

(Photo: Ambre Nolen)
Abdellatif Laâbi est né en 1942, à Fès (Maroc). Il fonde en 1966 la revue Souffles, qui jouera un grand rôle dans le renouvellement de la pensée et de la création culturelle au Maghreb. Poète, romancier, dramaturge, il est aussi traducteur en français de la poésie arabe moderne.

Son identidé de poète lui fait vivre l'insoumission. Elle lui vaut d'être emprisonné dans son pays de 1972 à 1980. Il vît en France depuis 1985. Pour lui, la poésie doit renouer avec l'oralité, retrouver son public, cesser de se lamenter sur sa marginalité. Son combat: être un homme libre. N'attend rien de la vie, va plutôt à sa rencontre. Ouvrages: Aux éditions Denoël, Chroniques de la citadelle d'exil, lettres de prison (1983); aux éditions de La Différence, Le soleil se meurt, poèmes (1992); Le Spleen de Casablanca, poèmes (1996); Poèmes

périssables, (2000) Rimbaud et Shéhêrazade, théâtre (2000); aux éditions Paroles d'aube Un continent humain, entretiens, poèmes (1997) Fragments d'une genèse oubliée, poème (1998); aux éditions Seuil-Jeunesse L'Orange bleue, poèmes (1995). Son roman Le Chemin des ordalies a été traduit en plusieurs langues, dont l'anglais, sous le titre Rue du Retour (Readers International Inc., Londres, 1989).

\title{
Littérature et éthique
}

\section{Abdellatif Laâbi}

$\mathrm{L}$ e mot d'éthique peut paraître excessif quand on le rapporte à la littérature. Après tout, si on jette un regard lucide sur l'histoire de la littérature, on découvre que cette dernière n'est pas, comme on voudrait le croire, le domaine privilégié de la vertu. C'est un champ de l'activité humaine presque comme les autres, où les contradictions et les passions battent leur plein, avec leur cortège d'intérêts, d'enjeux de pouvoir et de séduction, d'amour 
et de haine, de jalousie et de complicité, de bonté et de bassesse, de vérité et d'erreur, de volonté de puissance et de don magnifique de soi. Et, dans ce vaste théâtre de la conscience humaine, l'écrivain n'est pas toujours le roc solide qu'on croit, le vigile ne connaissant pas le sommeil, la conscience aiguë à laquelle rien n'échappe. Il est aussi un être comme vous et moi, avec sa force et sa faiblesse, sa fragilité et ses limites, ses connaissances et ses lacunes, ses dons de vision et ses oeillères, sa folie d'espérance et ses accès de désespoir, son amour de la vie et son désarroi face à l'énigme de la mort, et puis ses besoins les plus simples, les plus universels, d'amour, de paix et, pourquoi pas, de bonheur.

Mais, une fois que nous avons "humanisé" ainsi le personnage au point de le banaliser, se pose une autre question, celle ayant trait à sa responsabilité particulière à partir du moment où il a choisi l'activité qui est la sienne et non une autre. Après tout, et pour ne donner qu'un exemple, celui qui a opté pour la médecine sait qu'il va devoir prêter le serment d'Hippocrate avant d'exercer. Et c'est la fidélité à ce serment qui va déterminer, en bien ou en mal, le déroulement de son exercice.

On me rétorquera que l'exemple est mal choisi car l'écrivain n'est astreint, lui, à aucun serment, à aucun engagement préalable. L'écriture est un acte libre qui lui permet de s'adresser aux autres et de leur proposer sa lecture de la réalité humaine. Les lecteurs sont à leur tour libres d'accueillir cette proposition ou de s'adresser ailleurs.

L'objection paraît pertinente de prime abord. Mais elle ne résiste pas à l'analyse de ce qui fait la spécificité dẹ la pratique littéraire. Car, s'il y a une éthique de la littérature, elle ne réside certainement pas dans une injonction morale qui viendrait s'imposer à elle de l'extérieur. Non que cette injonction ne se présente pas. On peut même dire qu'elle fut la règle dans une phase relativement récente de l'histoire littéraire, et plus particulièrement des littératures africaines. L'attitude qui prévalait partait d'une appréciation contradictoire du rôle social de la littérature et du statut de l'écrivain. Ce dernier était perçu comme un porteur de conscience, un guide, voire un prophète. Mais il ne pouvait remplir ces divers offices que dans la mesure où il s'inscrivait dans un projet social dont l'élaboration et la mise en pratique relevaient, elles, des idéologues et des politiciens chevronnés. L'engagement de l'écrivain n'avait de validité que s'il adhérait à la ligne d'un parti, d'un mouvement de masse ou d'un appareil d'Etat, s'il s'intégrait dans ce que l'on appelait l'intellectuel collectif.

La plupart des écrivains africains ont traversé cette expérience. Certains y ont laissé une partie de leur âme. D'autres l'ont vécue comme une épreuve du feu qu'il fallait assumer pour partager réellement les souffrances, les aspirations et les combats de leur peuple. Quoi qu'il en soit, la phase historique concernée par notre analyse ne laissait nulle place à la marginalité. Le je devait être nécessairement un nous. La culture était une arme de combat, mais une arme d'appoint. Le primat du politique était incontestable.

Les malentendus qui ont entouré et entourent encore la question de l'engagement viennent assurément de là. Ils traduisent une vraie problématique. Au moment où il écrit, l'écrivain peut-il ou doit-il faire taire en lui le citoyen concerné par les affaires de la Cité? Comment une activité aussi solitaire que l'écriture peut-elle être solidaire ? L'adhésion à 
une cause, quelque généreuse qu'elle soit, ne risque-t-elle pas d'émousser chez l'écrivain l'esprit critique, le sens du relatif et la dialectique de la contradiction inhérente à toute conviction, toute pratique?

Je pense que ces questions resteront abstraites, ainsi que les réponses qu'on pourrait leur apporter, tant que l'on n'aura pas abordé la matière vivante, celle qui est au centre de notre réflexion, à savoir l'écriture. Mais il faudra l'aborder de l'intérieur, dans son processus d'élaboration et d'énonciation, dans ce corps à corps insolite que l'écrivain livre au silence têtu des mots. Dès lors, la question de l'éthique se présente sous un angle différent. D'injonction extérieure, elle devient intrinsèque à l'oeuvre. Ce qui va la déterminer, c'est l'attitude qui sera adoptée dans et face à l'écriture. Je veux parler du combat que l'écrivain mène sans cesse contre lui-même pour ne pas succomber à la facilité, aux rnodes, à la routine, et pour rejeter toute idée d'acquis "définitif" s'agissant de son travail. C'est ce niveau d'exigence qui l'amène à se remettre en question dès qu'il sent que son écriture s'assagit, se fige dans un moule, se stabilise dans son rapport à la langue, dans sa capacité à inventer des formes nouvelles.

Une telle attitude est aux antipodes de celle de l'écrivain qui construit un style et qui, dès qu'il croit l'avoir trouvé, s'en satisfait pour développer tranquillement sa carrière. Il se gardera alors de toute rupture pouvant déstabiliser le public qu'il a réussi à « fidéliser », comme on dit en termes marchands.

La première exigence d'ordre éthique est donc pour moi cet engagement sans calcul dans l'aventure de l'écriture, ce refus de la facilité, de toute concession aux vogues et au système littéraire dominant. L'acte d'écrire est une insoumission permanente dont le prix est connu: la marginalisation. Mais cette liberté souveraine n'est pas négociable, monnayable. C'est elle qui fonde, à mon avis, une certaine vérité à laquelle peut prétendre la parole.

$\mathrm{J}$ 'en viens maintenant à un deuxième niveau où se révèle le lien de l'écriture à l'éthique. Si le premier est intrinsèque, le second va plutôt de l'intérieur vers l'extérieur. Il découle donc du premier. Si l'écriture fonctionne pour moi selon une éthique, elle transmet les valeurs de cette dernière et impose à l'écrivain d'être en harmonie avec ses exigences dans l'ensemble de ses pratiques, sociales, intellectuelles, et de vie tout court.

Je sais bien que nous sommes là sur un terrain glissant. Il faut être aveugle pour ne pas constater qu'il existe dans de nombreux cas, parfois célèbres, un hiatus considérable entre l'écrivain et son oeuvre. J'ai mis personnellement beaucoup de temps à admettre cette réalité et à lire sans préjugés les oeuvres de certains écrivains talentueux mais peu respectables en tant qu'hommes. Si l'invective ne sert à rien dans ces cas-là, cela n'empêche pas de se battre à l'échelle individuelle contre l'hiatus, de rechercher non pas l'harmonie totale, mais au moins une cohérence entre les exigences de l'oeuvre et les exigences de vie. Car que vaudrait une oeuvre littéraire qui ne reposerait que sur le parâtre au lieu d'expliciter l'être ? Elle participerait du mensonge triomphant qui vise à entraîner la culture dans sa spirale et ouvrirait la voie à une nouvelle barbarie.

Finalement, il me semble que l'exigence qui se révèle dans l'écriture est celle qui détermine toutes les autres. C'est en se soumettant à elle que l'écrivain devient plus libre. 
$C^{\prime}$ est en acceptant ses rigueurs qu'il se forge la sienne. C'est en allant quotidiennement à son rendez-vous qu'il se rend présent à lui-même et au monde, qu'il reste à l'écoute des voix qui vont emprunter sa voix, qu'il reçoit la vie comme un don et s'empresse d'en restituer l'offrande.

La littérature ne gagne rien à s'éloigner de son credo humaniste. Au contraire, elle s'étiole et perd progressivement les facultés qui lui permettent d'être au coeur de l'aventure humaine, de se battre pour les belles raisons de vivre, d'aimer, d'espérer, et d'en appeler inlassablement au sursaut des consciences. Pour moi, l'écriture est avant tout un acte de dignité face à la comédie et à la tragédie humaines. Elle rend insupportables la bêtise, l'indifférence, la démission et le fanatisme. Ecole d'exigence et de liberté, c'est un des biens les plus précieux de l'homme. 